Check for updates

Cite this: RSC Adv., 2017, 7, 23550

\title{
Asymmetric isomerization: an efficient strategy to tune the electrical resistive memory behaviors of functional polyimides containing $N$ - phenylcarbazole moieties $\uparrow$
}

\author{
Nanfang Jia, (D) ab Guofeng Tian, (DD ab Shengli Qi, ${ }^{\text {ab }}{ }^{\text {Junhao Cheng, }}{ }^{\text {ab }}$ \\ Xiaodong Wang (D) ab and Dezhen $\mathrm{Wu}^{\mathrm{ab}}$
}

To elucidate the influence of spatial structure on memory behavior, three isomeric polyimides (PIs), PI-3,6DAPCz-6FDA, PI-3,4'-DAPCz-6FDA, and PI-2',4'-DAPCz-6FDA, for electrical memory applications are designed and synthesized. Current-voltage characteristics indicate that the three isomeric Pls display vastly deviating memory effects. PI-3,6-DAPCz-6FDA and PI-3,4'-DAPCz-6FDA both show volatile static random access memory (SRAM) behavior, while PI-2',4'-DAPCz-6FDA exhibits a non-volatile write once read many times (WORM) memory characteristic. The mechanisms associated with the memory effect were analyzed based on molecular simulation results. PI-3,6-DAPCz-6FDA possesses the best structural coplanarity, which greatly facilitates its charge transfer (CT) and back CT process, leading to the volatile SRAM feature. However, PI-2', 4'-DAPCz-6FDA exhibits more torsional structures, which consequently prohibits the back CT process, explaining its non-volatile behavior. Additionally, the three isomeric PIs show fast transient responses. The results show the importance of the spatial structure on polymer memory and the viability of adjusting memory types by isomerization.

\begin{abstract}
Received 24th March 2017 Accepted 24th April 2017

DOI: $10.1039 / \mathrm{c} 7 \mathrm{ra03454g}$

rsc.li/rsc-advances
\end{abstract}

Mechanisms related to the electrical bistability of these PIs containing D-A structure have also been extensively discussed, and charge transfer complex (CTC) formation ${ }^{4,5}$ is the mostcommonly accepted interpretation. To vary the charge transfer (CT) effect and then the memory behavior of the functional PIs, previous works mainly focus on the design and synthesis of novel electroactive PIs by introducing various different electron donors, including triphenylamine (TPA), ${ }^{22}$ ferrocene, ${ }^{23}$ carbazole $(\mathrm{Cz}){ }^{24}$ porphyrin, ${ }^{25}$ thiophene, ${ }^{14}$ selenophene ${ }^{14}$ and triphenylethylene,$^{15}$ to explore the effect of their electron-donating ability on the memory behaviors. However, due to the limited amount of electron-donating species and the difficulty in designing new species, this strategy has hit a bottleneck recently. As an alternative, it is well known that spatial effect usually has significant impact on the electronic structure of the organic electroactive species. Thus, it is considered that altering the spatial position of an electron-donating species in a functional PI would probably change the electronic CT process and might provide an effective approach to adjust the electrical memory features. Isomerization might be the simplest way to alter the spatial structure of a functional PI, but was rarely investigated.

Upon this consideration, three isomeric PIs for memory application are designed and synthesized in this work though the polycondensation of 6FDA with three isomeric DAPCz. The synthesized PIs, abbreviated as PI-3,6-DAPCz-6FDA, PI-3,4'-
${ }^{a}$ State Key Laboratory of Chemical Resource Engineering, Beijing University of Chemical Technology, Beijing 100029, China. E-mail: qisl@mail.buct.edu.cn; Tel: +86-10-6442-4654

${ }^{b}$ Changzhou Institute of Advanced Materials, Beijing University of Chemical Technology, Changzhou 213164, Jiangsu, China

$\dagger$ Electronic supplementary information (ESI) available. See DOI: $10.1039 / \mathrm{c} 7 \mathrm{ra03454 \textrm {g }}$ 
DAPCz-6FDA and PI-2', $4^{\prime}$-DAPCz-6FDA, have exactly the same chemical composition but vastly different spatial configuration, as shown in Scheme 1. Isomerization has significantly altered the relative spatial location of the electron-donating DAPCz unit and the electron-withdrawing 6FDA unit, which consequently should have considerable influence on the electronic structures of the synthesized PIs. Thus, it is expected that the CT process will be greatly altered, leading to varied electrical memory behaviors. Current-voltage $(I-V)$ characteristics of the memory devices with the structure of ITO/PI/Al indicates that the PI-3,6DAPCz-6FDA and PI-3,4'-DAPCz-6FDA manifest volatile SRAM behavior with different retention time (i.e., $1 \mathrm{~min}$ and $5 \mathrm{~min}$ ), while a non-volatile WORM behavior for the PI- $2^{\prime}, 4^{\prime}-\mathrm{DAPCz}$ 6FDA was observed. Besides, the isomeric PIs exhibit short electrical transient response time, i.e., $60 \mathrm{~ns}, 80 \mathrm{~ns}$, and $110 \mathrm{~ns}$ for the PI-3,6-DAPCz-6FDA, PI-3,4'-DAPCz-6FDA, and PI-2' $4^{\prime} 4^{\prime}$ DAPCz-6FDA, respectively. Optical and electrochemical measurements and molecular simulation calculated on the DFT B3LYP/6-31G(d) theory level were performed to clarify the CT process and the corresponding mechanisms of the distinct memory performances. The conclusion indicates the significant influence of spatial structure, and the accessibility to tune PI memory behaviors through structural isomerization.

\section{Experimental section}

\subsection{Materials}

Carbazole (99\%) and copper nitrate hydrate $\left(\mathrm{Cu}\left(\mathrm{NO}_{3}\right)_{2} \cdot 2.5 \mathrm{H}_{2} \mathrm{O}\right)$ were purchased from Sinopharm Chemical Reagent Co. Ltd. and used as received. $N$-phenylcarbazole $(\mathrm{PCz})$ was bought from Sigma-Aldrich and used without further purification. Hydrazine monohydrate $\left(\mathrm{N}_{2} \mathrm{H}_{4} \cdot \mathrm{H}_{2} \mathrm{O}\right)$ and 6FDA were got from Alfa Aesar and used as received. 1-Fluoro-2,4-dinitrobenzene was purchased from Aladdin Industrial Inc. 1-Fluoro-4nitrobenzene was received from Energy Chemical Reagent Co. Ltd. The $10 \%$ palladium on activated carbon was obtained from TCI. The $m$-cresol was purchased from Sinopharm Chemical Reagent Co. Ltd. and purified by distillation prior to use. Methanol, ethanol, $\mathrm{N}, \mathrm{N}$-dimethylformamide (DMF), $\mathrm{N}, \mathrm{N}$-dimethylacetamide (DMAc), and dimethyl sulfoxide (DMSO) were produced by Beijing Chemical Works and directly used without further purification.

\subsection{Instruments and measurements}

${ }^{1} \mathrm{H}$ NMR spectra of the synthesized compounds were tested on a Bruker AV400 spectrometer using tetramethylsilane as an internal standard at the resonant frequency of $400 \mathrm{MHz}$. FT-IR spectra were measured by a Bruker Tensor 27 infrared spectrophotometer. The weight average molecular weight $\left(M_{\mathrm{w}}\right)$ and number average molecular weight $\left(M_{\mathrm{n}}\right)$ of the synthesized isomeric PIs were evaluated by size exclusion chromatography (SEC) on a Waters 1525-2414 system calibrated by monodispersed polystyrene standard samples. DMF containing

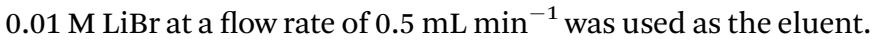
Elemental analysis was analyzed on a varioELcube elemental analyzer. The thermal properties were characterized by differential scanning calorimetry (DSC) and thermogravimetric analysis (TGA). DSC and TGA were conducted on a TA Q20 system at the heating rate of $20^{\circ} \mathrm{C} \mathrm{m^{-1 }}$ and a TA Q50 instrument at the heating rate of $10{ }^{\circ} \mathrm{C} \mathrm{min}^{-1}$, respectively. The thickness, film morphology and surface roughness were characterized by atomic force microscopy (AFM) with a Nanoscope 3D Controller in tapping mode. The optical characterization, ultraviolet-visible (UV/vis) absorption spectrum, was carried out on a Shimadzu UV-2550 spectrophotometer. The electrochemical measurement, cyclic voltammetry (CyV), was performed on a CHI660D electrochemical workstation in a three-electrode cell under nitrogen atmosphere. The polyimide layer on the platinum electrode were scanned (scan rate: $100 \mathrm{mV} \mathrm{s}^{-1}$ ) in a solution of tetrabutylammonium tetrafluoroborate $\left(n-\mathrm{Bu}_{4} \mathrm{BF}_{4}\right)$ in dry acetonitrile $(0.1$ $\mathrm{M}) . \mathrm{Ag} / \mathrm{AgCl}$ electrode is used as the reference electrode and platinum net as the auxiliary electrode. For calibration, we used ferrocene as the external reference $(0.38 \mathrm{~V}$ vs. $\mathrm{Ag} / \mathrm{AgCl})$. The electrical bistability and operation stability of the memories were characterized on a Keithley 4200 SCS semiconductor parameter analyzer equipped with a Micromanipulator PW-600 probe station (Advanced Technology Co. Ltd, Hong Kong).

\subsection{Synthesis and characterization of the isomeric polyimides}

Scheme 1 displays the chemical structures of the three $\mathrm{N}$ phenylcarbazole-based isomeric diamine monomers synthesized in this work, i.e., $N$-phenyl-3,6-diaminocarbazole (3,6DAPCz), $\quad N$-( $4^{\prime}$-aminophenyl)-3-aminocarbazole $\quad\left(3,4^{\prime}\right.$-DAPCz $)$ and $N$ - $\left(2^{\prime}, 4^{\prime}\right.$-diaminophenyl $)$-carbazole $\left(2^{\prime}, 4^{\prime}\right.$-DAPCz $)$, and the

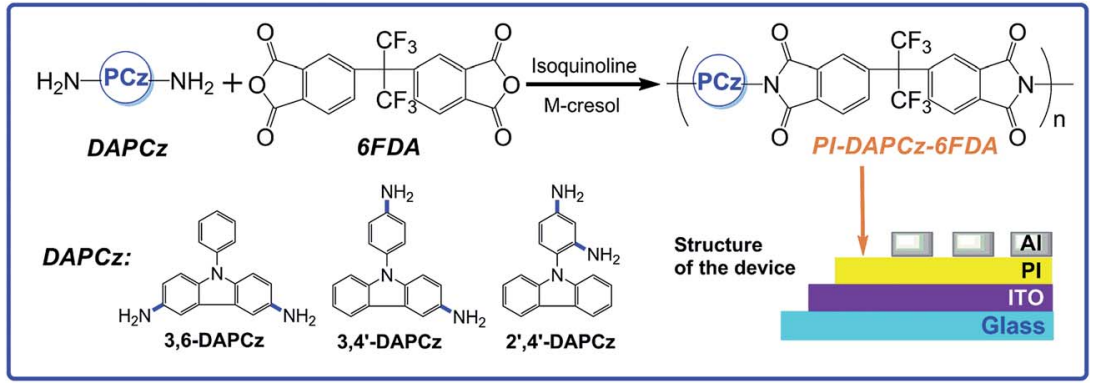

Scheme 1 Synthesis route of the isomeric polyimides containing isomeric $N$-phenylcarbazole moieties and illustrative configuration of the sandwich memory devices. 
synthetic route of target isomeric PIs. Detailed synthetic route, experiment condition and characterization of the isomeric diamines are described in the ESI (see Scheme S1 $\dagger$ ).

The isomeric PIs were synthesized through the condensation polymerization of the isomeric diamines with the wide-used dianhydride, 6FDA, by the typical two-step chemical imidization process. Taking the synthesis of the PI-3,6-DAPCz-6FDA as an example. In a $25 \mathrm{~mL}$ round-bottom flask, 6FDA (0.4442 g, 1 mmol) was added in one portion to a mixture of $3,6-\mathrm{DAPCz}$ $(0.2733 \mathrm{~g}, 1 \mathrm{mmol})$ and $m$-cresol $(7 \mathrm{~mL})$. The solution was stirred by a magnetic stirrer at room temperature for $2 \mathrm{~h}$ under nitrogen atmosphere to form a precursor of polyamic acid (PAA). Then the dehydrating agent, isoquinoline $(1 \mathrm{~mL})$ was added to the system to imidize the PAA to PI. After being heated at $190{ }^{\circ} \mathrm{C}$ for $12 \mathrm{~h}$, the resulting system was poured into a mass of methanol under stirring to get the crude product as a precipitate, which was then collected by filtration, washed thoroughly with methanol, and subsequently dried in vacuum oven at $100{ }^{\circ} \mathrm{C}$ to get the final PI as a yellow powder $(0.574 \mathrm{~g}$, $84 \%$ in yield). The PI-3, $4^{\prime}$-DAPCz-6FDA and PI-2 ${ }^{\prime}, 4^{\prime}$-DAPCz-6FDA were prepared according to a similar procedure with the final yield of $86 \%$ and $79 \%$, respectively.

For the PI-3,6-DAPCz-6FDA: SEC $\left(\mathrm{g} \mathrm{mol}^{-1}\right): M_{\mathrm{n}}=9.2 \times 10^{4}$, $M_{\mathrm{w}}=1.2 \times 10^{5}, M_{\mathrm{w}} / M_{\mathrm{n}}=1.34$. FT-IR $\left(\mathrm{KBr}, \mathrm{cm}^{-1}\right): 1784,1726$, 1356, 719 (see Fig. 1(a)). ${ }^{1} \mathrm{H}-\mathrm{NMR}$ (DMSO-d $\left.{ }_{6}, 400 \mathrm{MHz}\right), \delta(\mathrm{ppm})$ : 8.25-8.31 (m, 2H), 8.18 (s, 2H), 7.98 (s, 2H), 7.79 (s, 2H), 7.69$7.75(\mathrm{~m}, 4 \mathrm{H}), 7.51-7.58(\mathrm{~m}, 5 \mathrm{H})$ (see Fig. 1(b)). Elemental analysis: calc. for PI-3,6-DAPCz-6FDA (\%), C, 57.76; H, 3.05; N, 7.48. Found: C, 56.78; H, 2.92; N, 7.52.

For the PI-3,4'-DAPCz-6FDA: SEC $\left(\mathrm{g} \mathrm{mol}^{-1}\right): M_{\mathrm{n}}=4.7 \times 10^{4}$, $M_{\mathrm{w}}=5.7 \times 10^{4}, M_{\mathrm{w}} / M_{\mathrm{n}}=1.22$. FT-IR $\left(\mathrm{KBr}, \mathrm{cm}^{-1}\right): 1783,1725$, 1354, 718 (see Fig. 1(a)). ${ }^{1} \mathrm{H}-\mathrm{NMR}$ (DMSO-d $\left.{ }_{6}, 400 \mathrm{MHz}\right), \delta$ (ppm): $8.34(\mathrm{~s}, 1 \mathrm{H}), 8.24-8.26(\mathrm{~m}, 3 \mathrm{H}), 8.02(\mathrm{~d}, 2 \mathrm{H}, J=6.1 \mathrm{~Hz}), 7.89(\mathrm{~s}$, $2 \mathrm{H}), 7.81-7.86(\mathrm{~m}, 4 \mathrm{H}), 7.60(\mathrm{~d}, 1 \mathrm{H}, J=7.9 \mathrm{~Hz}), 7.52(\mathrm{~s}, 3 \mathrm{H}), 7.36$ (s, 1H) (see Fig. 1(b)). Elemental analysis: calc. for PI-3,6-DAPCz6FDA (\%), C, 57.76; H, 3.05; N, 7.48. Found: C, 56.59; H, 2.82; N, 7.57 .

For the PI-2' ${ }^{\prime} 4^{\prime}$-DAPCz-6FDA: SEC $\left(\mathrm{g} \mathrm{mol}^{-1}\right): M_{\mathrm{n}}=2.7 \times 10^{4}$, $M_{\mathrm{w}}=5.4 \times 10^{4}, M_{\mathrm{w}} / M_{\mathrm{n}}=2.01$. FT-IR $\left(\mathrm{KBr}, \mathrm{cm}^{-1}\right): 1786,1732$, 1355, 720 (see Fig. 1(a)). ${ }^{1} \mathrm{H}-\mathrm{NMR}$ (DMSO-d $\left.{ }_{6}, 400 \mathrm{MHz}\right), \delta(\mathrm{ppm})$ : 8.17-8.26 (m, 2H), 8.05-8.09 (m, 2H), 7.92-7.95 (m, 2H), 7.85 (s,
2H), $7.78(\mathrm{~s}, 2 \mathrm{H}), 7.68(\mathrm{~s}, 1 \mathrm{H}), 7.58(\mathrm{~s}, 1 \mathrm{H}), 7.44(\mathrm{~s}, 1 \mathrm{H}), 7.29$ (s, $2 \mathrm{H}$ ), 7.15 (d, 2H, $J=10.5 \mathrm{~Hz}$ ) (see Fig. 1(b)). Elemental analysis: calc. for PI-3,6-DAPCz-6FDA (\%), C, 57.76; H, 3.05; N, 7.48. Found: C, 56.63; H, 2.71; N, 7.57.

Here, it should be mentioned that, due to the asymmetric structures of the $3,4^{\prime}-\mathrm{DAPCz}$ and $2^{\prime}, 4^{\prime}$-DAPCz, the synthesized isomeric PIs, PI-3, $4^{\prime}$-DAPCz-6FDA and PI- $2^{\prime}, 4^{\prime}$-DAPCz-6FDA, are bound to have stereo-irregular configuration structures (i.e., isotactic, syndiotactic, or atactic) in their long polymer chains, as illustrated in Scheme S2. $\dagger$ However, currently, it is practically infeasible for us to control their stereo-regularity during our condensation polymerization process. Thus, the steric regularity of the PI was not discussed. Whereas, it is suggested that the PIs synthesized in this work were most probably atactic, since the chemical reactivity of the two amino groups in both $3,4^{\prime}$-DAPCz and $2^{\prime}, 4^{\prime}$-DAPCz do not exhibit vast deviations as evidenced by the two close amino proton chemical shifts in the ${ }^{1} \mathrm{H}-\mathrm{NMR}$ spectra (see Fig. S2 and S3†), and condensation polymerization normally yields random polymer with no regularity. Besides, DSC measurements (see Fig. 2(b)) detect only one glass transition temperature $\left(T_{\mathrm{g}}\right)$ for each PI, probably implying atactic regularity.

The synthesized isomeric PIs exhibit considerably good solubility at room temperature in common organic solvents, such as $N$-methyl-2-pyrrolidone (NMP), DMAc, DMF, DMSO, $m$ cresol, tetrahydrofuran (THF) and chloroform. A qualitative evaluation indicates that the isomeric PIs are readily dissolved in the above solvents to give a homogenous solution with concentration of $30 \mathrm{mg} \mathrm{mL}^{-1}$, providing desirable feasibility for memory device fabrications via the solution process. Here, it is suggested that the asymmetric structures of the PI-3, $4^{\prime}-\mathrm{DAPCz}-$ 6FDA and PI- $2^{\prime}, 4^{\prime}$-DAPCz-6FDA play an important positive role in help promoting their solubility.

The thermal performances of the synthesized isomeric PIs were characterized by DSC and TGA measurements. As displayed in Fig. 2(a), the 5\% weight-loss temperatures and $10 \%$ weight-loss temperatures of the PI-3,6-DAPCz-6FDA, PI-3,4'DAPCz-6FDA and PI-2', $4^{\prime}$-DAPCz-6FDA in $\mathrm{N}_{2}$ were all recorded to be over $530{ }^{\circ} \mathrm{C}$. The high decomposition temperature indicates high-temperature stability of the titled PIs, due to their rigid aromatic main chain structure. And, as shown in Fig. 2(b), the

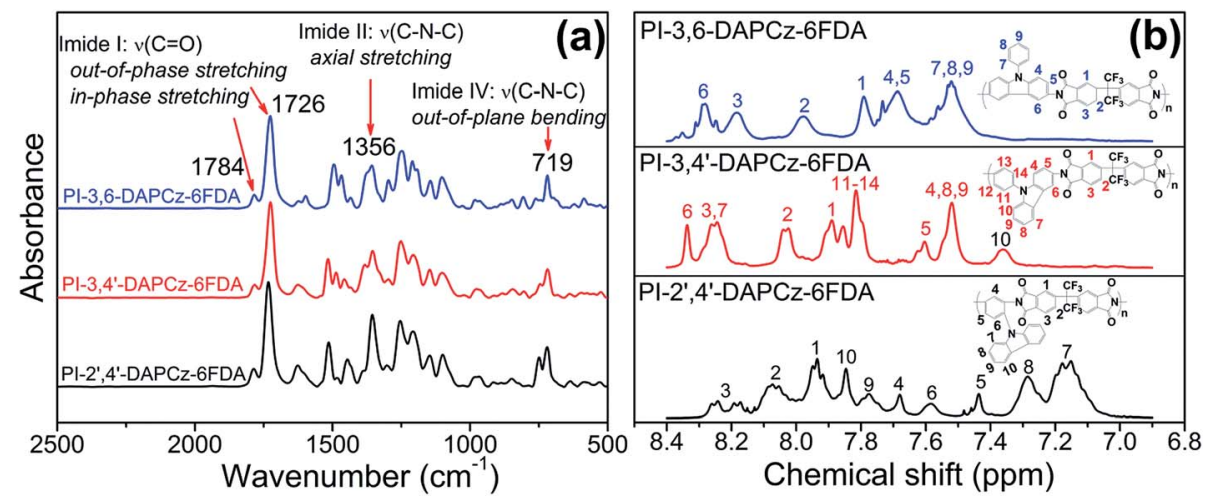

Fig. 1 (a) FT-IR spectra of the synthesized isomeric Pls; and (b) ${ }^{1} \mathrm{H}$ NMR spectra of the isomeric Pls measured in DMSO-d 6 . 

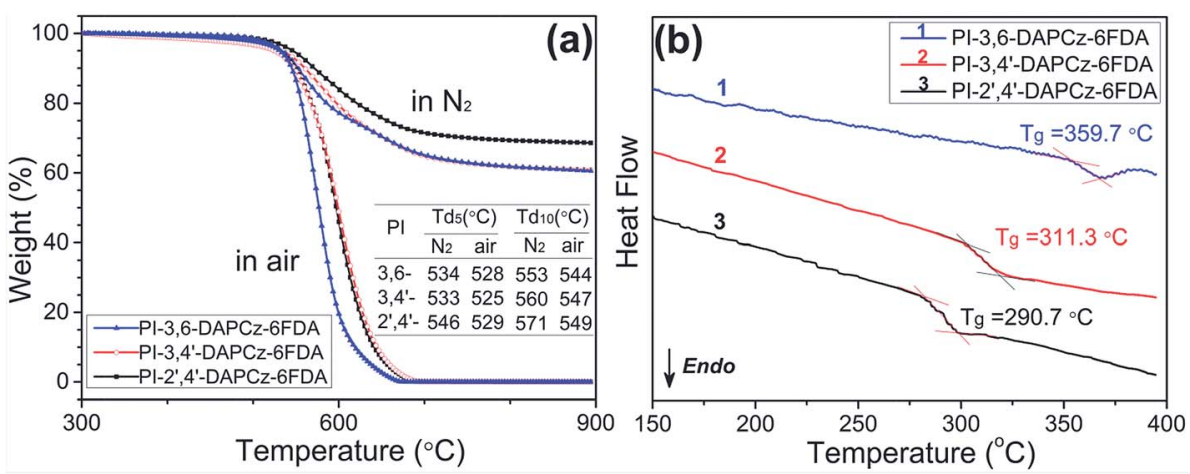

Fig. 2 (a) TGA curves of the isomeric PIs measured in both nitrogen and air atmosphere at a heating rate of $10^{\circ} \mathrm{C} \mathrm{min}{ }^{-1}$; (b) DSC curves of the synthesized isomeric Pls recorded in nitrogen at a heating rate of $20{ }^{\circ} \mathrm{C} \mathrm{min}{ }^{-1}$.

$T_{\mathrm{g}}$ of the PI-3,6-DAPCz-6FDA, PI-3,4'-DAPCz-6FDA and PI-2', $4^{\prime}-$ DAPCz-6FDA were recorded to be as high as $359.7,311.3$ and $290.7^{\circ} \mathrm{C}$, respectively. The $T_{\mathrm{g}}$ of the PI-3, $4^{\prime}$-DAPCz-6FDA and PI$2^{\prime}, 4^{\prime}$-DAPCz-6FDA are clearly degraded, as compared with PI-3,6DAPCz-6FDA. This is ascribed to the asymmetric and rather twisted chain structure, together with the decrease of the rigid carbazole unit in the main chain after isomerization. In addition, the char yield of the isomeric PIs in $\mathrm{N}_{2}$ atmosphere all exceeded $60 \%$ under $900{ }^{\circ} \mathrm{C}$, which is supposed to be resulted from the high content of the aromatic components. The good thermal performances will ensure the isomeric PIs adequate heat-resistance stabilities in real memory applications.

\subsection{Fabrication of the memory device}

The sandwich device was fabricated on the ITO-coated glass, with a typical configuration of ITO/PI/Al. The conductive ITO layer acted as the bottom electrode and $\mathrm{Al}$ as the top electrode. The ITO-coated glass was pre-cleaned under ultrasonication with isopropanol, ethanol, and chloroform and then dried by a $\mathrm{N}_{2}$ gun prior to use. Then, the DMAc solutions of the synthesized isomeric PIs with a concentration of $30 \mathrm{mg} \mathrm{mL}^{-1}$, filtered by PTFE membrane, were dropped onto the pre-cleaned ITO conductive substrate and spin-coated at $1500 \mathrm{rpm}$ for $60 \mathrm{~s}$ to serve as the active layer. Afterwards, the resulted PI films were dried at $120{ }^{\circ} \mathrm{C}$ for $5 \mathrm{~h}$ in a vacuum oven to remove the trace amount of solvent. Finally, an Al electrode with a thickness of about $100 \mathrm{~nm}$ was evaporated onto the PI surfaces by vacuum evaporation. The shape of the Al electrode was controlled by the shadow mask. Scheme 1 displays the schematic configuration of the fabricated memory devices.

The morphology of the polyimide active layer was characterized by AFM and shown in Fig. S4. $\dagger$ The thickness of the films is all around $50 \mathrm{~nm}(49.5 \mathrm{~nm}, 48.4 \mathrm{~nm}$ and $48.1 \mathrm{~nm}$ for PI-3,6DAPCz-6FDA, PI-3,4'-DAPCz-6FDA and PI-2', $4^{\prime}$-DAPCz-6FDA, respectively), which can eliminate the effect of different thickness on memory behavior. The root-mean-square roughness $\left(R_{\mathrm{q}}\right)$ of PI-3,6-DAPCz-6FDA, PI-3, $4^{\prime}$-DAPCz-6FDA and PI-2' ${ }^{\prime}{ }^{\prime}-$ DAPCz-6FDA films is $0.758 \mathrm{~nm}, 0.781 \mathrm{~nm}$ and $0.878 \mathrm{~nm}$, respectively, indicating fairly smooth surface, which can guarantee the carrier injection process and consequently lead to excellent stability of the memory behavior.

\subsection{Quantum chemical simulation}

Quantum chemical simulation was performed using the Gaussian 09 program package. To get a clear understanding of the electronic structures varied with the isomeric PIs structures, the favorable equilibrium geometries, Mulliken charges, molecular orbitals and energy levels were simulated by the density functional theory (DFT) and the B3LYP method with the 6-31G(d) basic set. For model simplicity, the basic units of the isomeric PIs were employed as the model compound for computation. All the structures were optimized on the AM1 level, HF level and B3LYP level in sequence. The stereoregularity (i.e., isotactic, syndiotactic, and atactic) practically existing in the asymmetric PI-2',4'-DAPCz-6FDA and PI-3,4'DAPCz-6FDA polymer chains were not considered during the DFT calculations due to the model complexity and computation unavailability.

\section{Results and discussion}

\subsection{Optical and electrochemical properties}

Fig. 3(a) displays the UV/vis absorption spectra of the isomeric PIs. The corresponding absorption maxima $\left(\lambda_{\max }\right)$ are listed in Table 1. All of the three PIs exhibit a prominent absorption peak at around $290 \mathrm{~nm}$, corresponding to the $\mathrm{n}-\pi^{*}$ transition occurring on the DAPCz unit. Besides, it is clear that all the three PIs display a broad absorbance band in the longer wavelength range of $300-350 \mathrm{~nm}$, which is suggested to be attributed to the intra- or inter-molecular $\pi-\pi^{*}$ transitions from the electron-donating DAPCz moieties to the electron-withdrawing 6FDA units, and the formation of CT complexes. However, it is noted that the absorbance of PI-3,6-DAPCz-6FDA and PI-3,4'DAPCz-6FDA in 300-350 $\mathrm{nm}$ are highly broadened with no distinct peaks, implying that the CT complexes formed in these two PIs are most likely to be weak and instable. In contrast, a clear absorption peak at around $321 \mathrm{~nm}$ is present for the PI$2^{\prime}, 4^{\prime}$-DAPCz-6FDA, suggesting the formation of much stronger and more stable CT complex. This difference provides a clear hint for the corresponding non-volatile and volatile memory behaviors of the isomeric polyimides, which will be discussed later. The onset wavelength, i.e., the absorption edge $\left(\lambda_{\text {edge }}\right)$ of the UV spectra of the isomeric PIs are extended to be about 

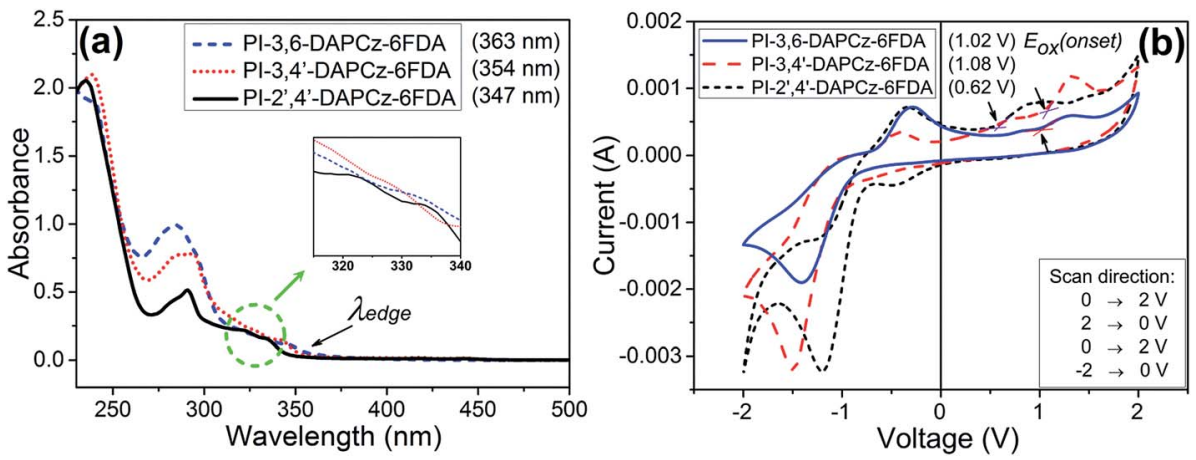

Fig. 3 (a) UV/vis absorption spectra of the three isomeric polyimides measured in THF and (b) cyclic voltammograms of the isomeric polyimide

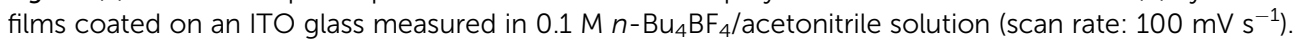

$363 \mathrm{~nm}$ (PI-3,6-DAPCz-6FDA), $354 \mathrm{~nm}$ (PI-3,4'-DAPCz-6FDA) and $347 \mathrm{~nm}$ (PI-2', $4^{\prime}$-DAPCz-6FDA), respectively, which give the $E_{\mathrm{g}}$ estimated by Plank equation $\left(E_{\mathrm{g}}=1240 / \lambda_{\text {edge }}\right)$ to be $3.42 \mathrm{eV}$, $3.50 \mathrm{eV}$ and $3.57 \mathrm{eV}$, respectively.

The electrochemical behaviors of the isomeric PIs are shown in Fig. 3(b) and summarized in Table 1. According to the CV spectra, an oxidation peak appeared in the positive scan of each PI. And the onset oxidation potential of these three PIs exhibited at $1.02 \mathrm{eV}$ (PI-3,6-DAPCz-6FDA), $1.08 \mathrm{eV}$ (PI-3,4'-DAPCz6FDA) and $0.62 \mathrm{eV}$ (PI-2 ${ }^{\prime}, 4^{\prime}$-DAPCz-6FDA), respectively. The HOMO energy levels of the isomeric PIs estimated from the onset of oxidation peak in CV were $-5.44 \mathrm{eV}$ (PI-3,6-DAPCz6FDA), $-5.52 \mathrm{eV}$ (PI-3,4'-DAPCz-6FDA) and -5.04 eV (PI-2', $4^{\prime}$ DAPCz-6FDA), respectively. The higher HOMO level of PI-2', $4^{\prime}$ DAPCz-6FDA indicates the stronger electron-donating ability of the $2^{\prime}, 4^{\prime}$-DAPCz species as compared with the other two isomers, 3,4'-DAPCz and 3,6-DAPC.

\subsection{Memory characteristics of the isomeric PIs}

The memory characteristics of the isomeric PIs were demonstrated by the $I-V$ curves of the ITO/PI/Al sandwich devices. As shown in Fig. 4(a), the PI-3,6-DAPCz-6FDA based device shows a volatile SRAM behavior with bi-directional accessibility.

During the first scan, the voltage ranges from 0 to $5 \mathrm{~V}$. A sharp increase in the current from $10^{-9}$ to $10^{-5}$ A was observed at the threshold voltage of about $2.5 \mathrm{~V}$, corresponding to the switching from the initial OFF state to the ON state of the device. After this transition, the device remains its ON state during the subsequent positive sweep (the $2^{\text {nd }}$ sweep) and then the negative sweep (the $3^{\text {rd }}$ sweep), indicating its "inerasable" characteristics that cannot be retrieved to the original OFF state by applying a reverse bias sweep. However, further characterization shows that the ON state of the device can be retained for only $1 \mathrm{~min}$, and relaxed readily to the OFF state after the power was turned off, indicating the actually volatile nature of the device. The $4^{\text {th }}$ voltage scan after turning off the power for over 1 min suggests that the device can be re-switched to and maintain the ON state during the followed $5^{\text {th }}$ and $6^{\text {th }}$ sweeps, indicating its "reprogrammable" characteristics. The $7^{\text {th }}$ (later the $\left.10^{\text {th }}\right)$ scan from 0 to $-5 \mathrm{~V}$ conducted after the power's turned off for over 1 min was also able to turn the device from the OFF state to the ON state following an essentially identical switching process, indicating that the current device can be operated bidirectionally with no polarity. These results indicate that the PI-3,6-DAPCz-6FDA based device exhibits SRAM behavior, with the typical volatile, reprogrammable and bi-directionally accessible characteristics. The volatile ON state of the device can be permanently retained by a refreshing voltage pulse of $1 \mathrm{~V}$ within $1 \mu$ s duration in every $5 \mu \mathrm{s}$ (named the rf trace), as shown in Fig. 4(a).

In addition to the memory behavior, other parameters including the ON/OFF current ratio, reading cycles, long-term retention stability and switching time also play crucial roles in determining the performances of the devices. Fig. 4(b) displays the stimulus effect of the continuous read pulses (magnitude: $1 \mathrm{~V}$, period: $2 \mu \mathrm{s}$, duration width: $1 \mu \mathrm{s}$ ) on the device. Both the ON and OFF states are readily survived up to $10^{8}$ continuous

Table 1 Optical and electrochemical properties of the synthesized isomeric polyimides

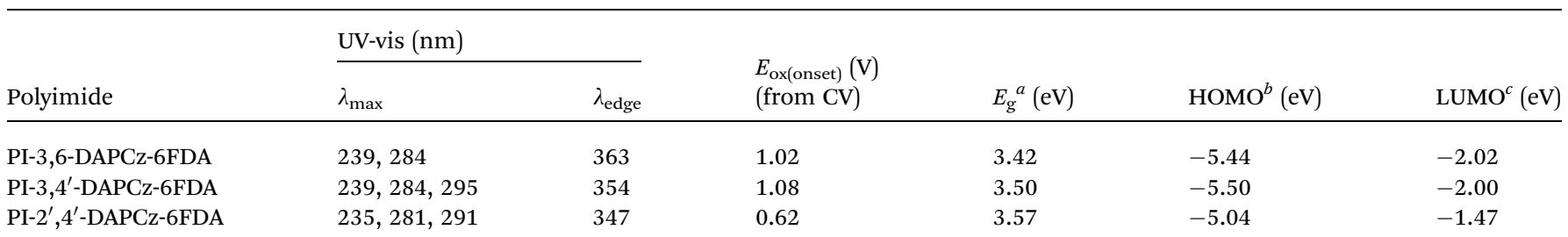

${ }^{a}$ Estimated from the UV-vis absorption edge wavelength $\left(\lambda_{\text {edge }}\right)$ using the Plank equation: $E_{\mathrm{g}}=1240 / \lambda_{\text {edge }}{ }^{b}$ The HOMO energy levels were determined from the $\mathrm{CV}$ onset ionization potential $\left(E_{\mathrm{ox}(\mathrm{onset})}\right)$ using ferrocene as the external reference (4.8 eV below the vacuum level): $\mathrm{HOMO}$ $=-\left[\left(E_{\text {ox }(\text { onset })}-E_{\text {ferrocene }}\right)+4.8\right](\mathrm{eV}) . E_{\text {ferrocene }}$ is determined to be $0.38 \mathrm{~V} v s . \mathrm{Ag} / \mathrm{AgCl} .{ }^{c}$ Determined from the equation: LUMO $=E_{\mathrm{g}}+\mathrm{HOMO}$. 

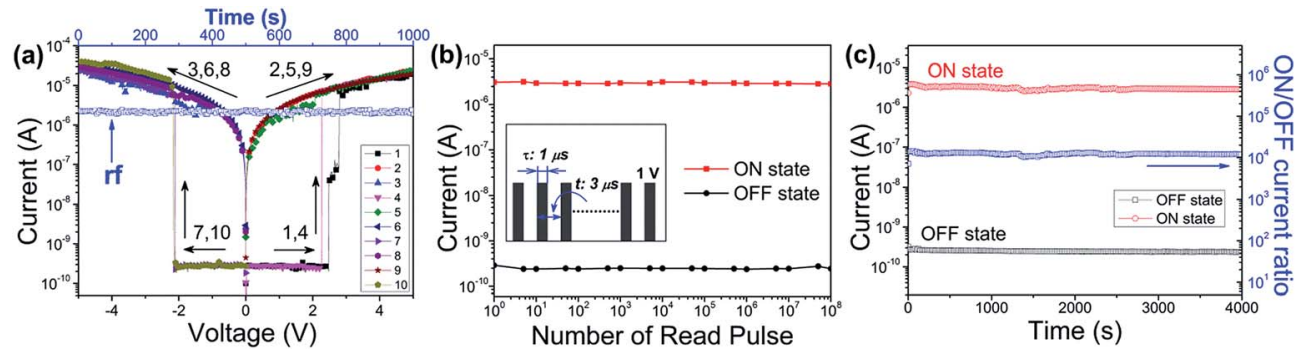

Fig. 4 (a) Current-voltage (I-V) characteristics (b) stimulus effect of read pulses on the ON and OFF states (c) retention time test on the ON and OFF states of the ITO/PI-3,6-DAPCz-6FDA/Al sandwich device.

read pluses. Fig. 4(c) displays the operation time effect on the PI-3,6-DAPCz-6FDA based memory device tested under a constant voltage of $1 \mathrm{~V}$. As displayed, no obvious current degradation was observed in both the ON state and OFF state and the ON/OFF current ratio was kept stably at around $10^{4}$ during the whole test, proving the outstanding long-term operation stability of the sandwich memory device.

The $I-V$ characteristic of the PI-3,4'-DAPCz-6FDA based device is shown in Fig. 5(a), which indicates a similar bidirectionally volatile SRAM memory behavior as the PI-3,6DAPCz-6FDA in Fig. 4(a). However, the ON state of PI-3,4'DAPCz-6FDA based device can sustain for $c a .5 \mathrm{~min}$ after the power's turned off, which is longer than that of PI-3,6-DAPCz6FDA. As well, the ON state can be retained by a refreshing voltage pulse of $1 \mathrm{~V}$ within $1 \mu \mathrm{s}$ duration in every $5 \mu \mathrm{s}$ (named the rf trace), as displayed in Fig. 5(a). The ON state and OFF state can keep stable in $10^{8}$ read pluses, as shown in Fig. 5(b). And long-term operation test in Fig. 5(c) reveals that the ON/ OFF current ratio of the PI-3, $4^{\prime}$-DAPCz-6FDA device is kept as high as around $10^{5}$.

Fig. 6(a) and (b) show the $I-V$ characteristics of the PI- $2^{\prime}, 4^{\prime}-$ DAPCz-6FDA based sandwich devices. A non-volatile WORM memory behavior was observed. As shown, during the first voltage scan from 0 to $4 \mathrm{~V}$, a sharp increase in current occurred at the voltage of $3 \mathrm{~V}$, turning the device from the OFF state of $10^{-9} \mathrm{~A}$ to the ON state of $10^{-4} \mathrm{~A}$. Further, the device is found to permanently sustain the ON state during the followed positive and negative scans, and cannot go back to the original OFF state, even after applying a reverse voltage sweep or the power's turned off for days. This fact indicates the non-volatile WORM behavior of PI-2', $4^{\prime}$-DAPCz-6FDA, which is entirely different from the volatile feature of the other two isomeric PIs. Additionally, the $I-V$ result shown in Fig. 6(b) indicates that the device could also be switched on when a negative bias scan was applied as the first sweep. Both the ON/OFF current ratio and the switching-on threshold voltage are similar in magnitude to those in Fig. 6(a), demonstrating the bi-directional switching and nonpolar features of the PI-2' $4^{\prime}$-DAPCz-6FDA based device. Long-term stability evaluation, as displayed in Fig. 6(c) and (d), manifests that the current device maintains an ON/OFF ratio at about $10^{4}$ and readily passes $10^{8}$ cycles of read pulses test, revealing excellent operation stability.

\subsection{Switching time}

As another key performance indicator, switching time can be used to characterize the fast-response property of the memory device. Fig. 7 displays the switching time and equilibrium time of the PI-3,6-DAPCz-6FDA, PI-3,4'-DAPCz-6FDA and PI-2' $4^{\prime}$ DAPCz-6FDA based memory devices at the voltage of $1 \mathrm{~V}$ and $4 \mathrm{~V}$. As shown in Fig. 7(a), (c) and (e), the memory devices initially stayed in the OFF state and cannot be switched to the ON state by a read pulse below the threshold voltage $(1.0 \mathrm{~V})$. Whereas, when a read pulse above the threshold voltage $(4.0 \mathrm{~V})$ was applied, the devices were all immediately switched to the ON state, as recorded in Fig. 7(b), (d) and (f). Meanwhile, no detectable electrical delay was observed, which indicates that the switching time of the memory devices is shorter than the limit of the pulse rising time, i.e., $20 \mathrm{~ns}$ for Keithley 4200 SCS semiconductor parameter analyzer. Besides, as can be seen, the memory devices can reach their stable ON states rapidly from the initial OFF states in an extremely short period (i.e., the
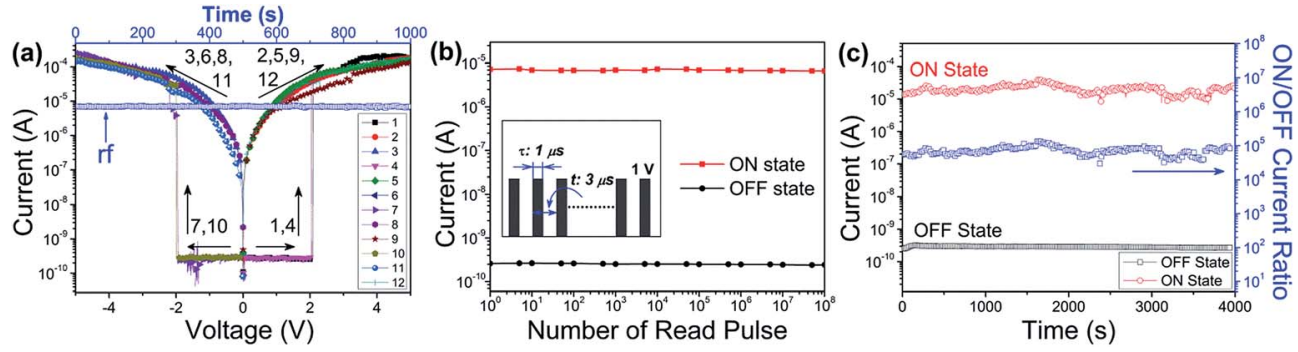

Fig. 5 (a) Current-voltage (I-V) characteristics (b) stimulus effect of read pulses on the ON and OFF states (c) retention time test on the ON and OFF states of the ITO/PI-3,4'-DAPCz-6FDA/Al sandwich device. 

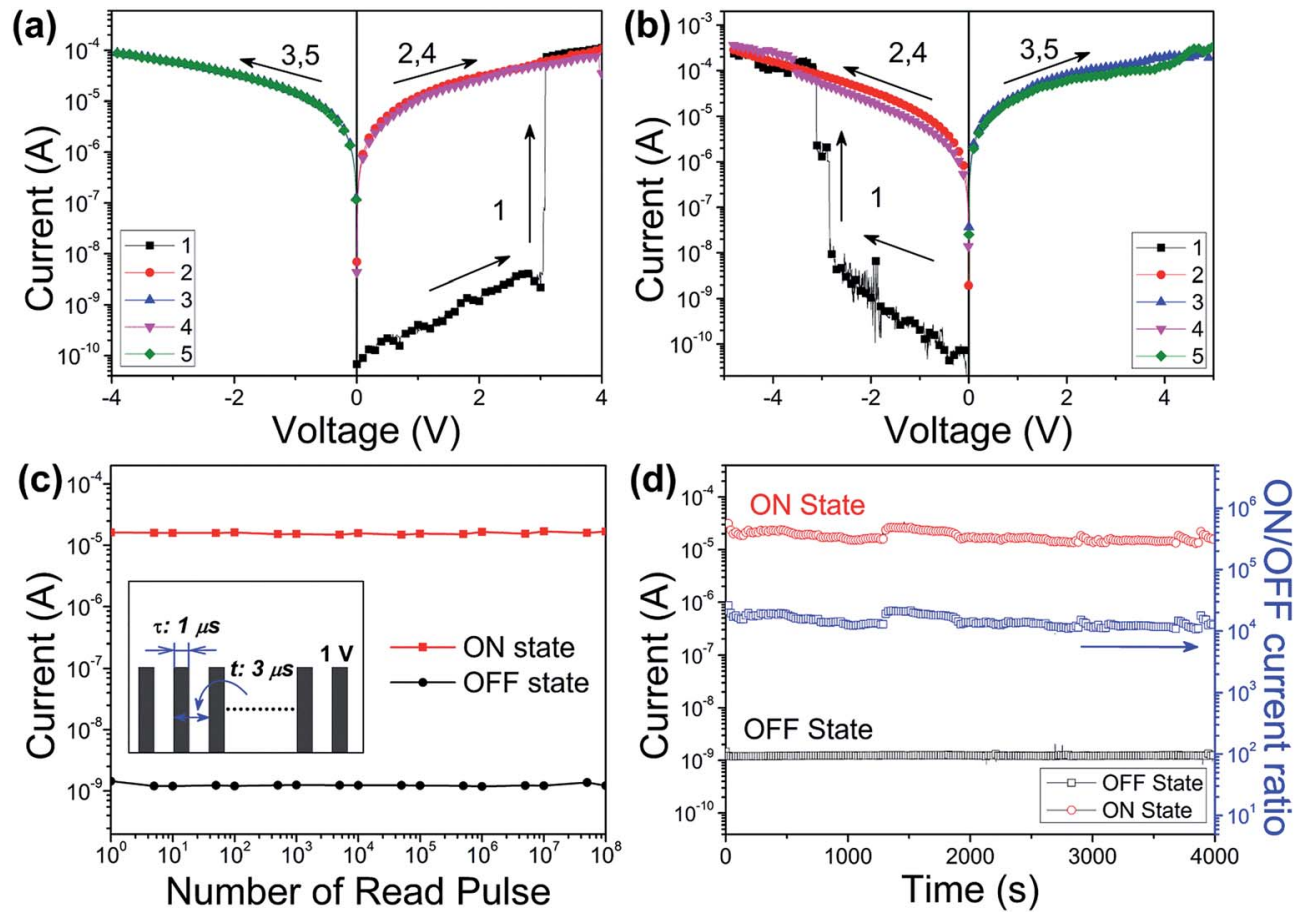

Fig. 6 Current-voltage $(I-V)$ characteristics of the ITO/PI-2', 4'-DAPCz-6FDA/Al sandwich device, the sweep sequence and direction are indicated by the number and arrow, respectively (first scan (a): 0 to $+4 \mathrm{~V}$; (b): 0 to $-5 \mathrm{~V}$ ); (c) stimulus effect of read pulses on the ON and OFF states of the ITO/PI-2', $4^{\prime}$-DAPCz-6FDA/Al device, the inset shows the pulse shapes in the measurement; (d) retention time test on the ON and OFF states of the ITO/PI-2', $4^{\prime}-$ DAPCZ-6FDA/Al device.
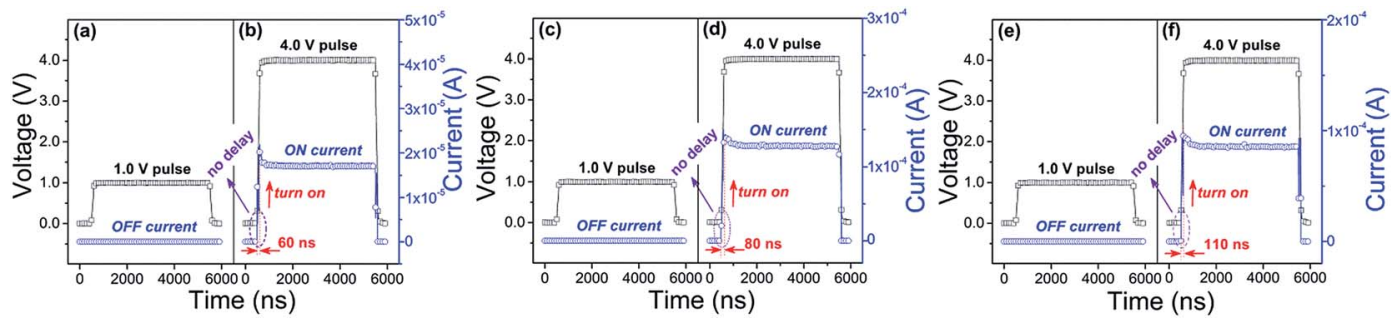

Fig. 7 The transient response of the device current vs. time below and above the switching-on threshold voltage. (a, c, and e) show the electrical current response of the PI-3,6-DAPCz-6FDA, PI-3,4'-DAPCz-6FDA and PI-2', 4'-DAPCz-6FDA devices initially in the OFF state. A voltage pulse $(1.0 \mathrm{~V})$ less than the switching-on threshold voltage is applied to confirm the low-conducting state of the devices. (b, $d$, and $f)$ show the electrical current response of the same PI-3,6-DAPCz-6FDA, PI-3,4'-DAPCz-6FDA and PI-2', 4'-DAPCz-6FDA devices under a voltage pulse (4.0 V) higher than the switching-on threshold voltages. The device responses to the applied voltage immediately with no distinguishable electrical delay and are transformed to their equilibrium high conductivity state in ca. 60, 80, $110 \mathrm{~ns}$, respectively.

equilibrium time): $60 \mathrm{~ns}$ for the PI-3,6-DAPCz-6FDA, $80 \mathrm{~ns}$ for the PI-3,4'-DAPCz-6FDA, and $110 \mathrm{~ns}$ for the PI-2', $4^{\prime}$-DAPCz6FDA. To summarize, it is clear that both the switching time and the equilibrium time of the fabricated memory devices based on the isomeric PIs are faster than that (about $1 \mu \mathrm{s}$ ) of the NAND (NOT/AND) flash memory based on traditional semiconductors, and comparable to the current widely-used DRAM and SRAM inorganic semiconductor memory electronics, of which the switching time is shorter than $10 \mathrm{~ns}$ and the access time ranges in 60-100 ns. These results suggest promising prospect of the isomeric PIs for the novel electronic memory applications characterized by large storage capacity and ultrafast access speed. Besides, it is considered that the ultrafast switching-on response in the isomeric PIs based devices is an indication of the occurrence of prompt charge transfer process inside the synthesized isomeric PIs.

\subsection{Quantum chemical simulation and switching mechanism}

In order to clarify the charge transition processes and the different memory behaviors occurring in the isomeric PIs, theoretical simulation was calculated on the density functional theory (DFT) with the B3LYP method on the 6-31G(d) theory level.

Table S1 $\uparrow$ summarizes the simulation results for the 3,6DAPCz, 3,4'-DAPCz, 2', $4^{\prime}$-DAPCz, 6FDA units and the model 
compounds of the three isomeric PIs. As expected, the three DAPCz units all exhibit higher HOMO energy levels (i.e., $-4.49 \mathrm{eV},-5.25 \mathrm{eV}$ and $-4.66 \mathrm{eV})$ than 6FDA $(-8.28 \mathrm{eV})$, indicating their strong tendency to donate electrons. Meanwhile, the 6FDA possesses a much lower LUMO energy level of $-2.98 \mathrm{eV}$, indicating its strong inclination to withdraw electrons. Fig. 8 displays the charge density isosurfaces of the calculated HOMO and LUMOs of the basic unit of the three isomeric PIs. As observed, when the isomeric DAPCz and 6FDA are covalently connected, the HOMO mainly locates on the electron-donating DAPCz unit, whereas the LUMOs exhibit clearly different distribution. For the PI-3,6-DAPCz-6FDA, the $\mathrm{LUMO} / \mathrm{LUMO}+1$ orbitals and the LUMO+2/LUMO+3 orbitals are apparently degenerated and symmetrically distributed on the 6FDA unit. While, for the asymmetric PI-3,4'-DAPCz-6FDA and PI- $2^{\prime}, 4^{\prime}$-DAPCz-6FDA, degeneration of the LUMO orbitals does not occur.

The PI-3,6-DAPCz-6FDA is taken as an example to illustrate the electronic transition process occurring in the isomeric PIs. As shown, the HOMO and LUMO+4 mainly distribute on the carbazole group of the electron-donating DAPCz unit, while the LUMO, LUMO+1, LUMO+2 and LUMO+3 entirely locate on the electron-withdrawing 6FDA unit. Once being excited under external voltage, electrons at the HOMO orbitals tend to transfer to different LUMOs, forming different excited states. And as a result of vertical excitation, electrons at the HOMO will be prone to transfer to the LUMO+4 directly due to the biggest

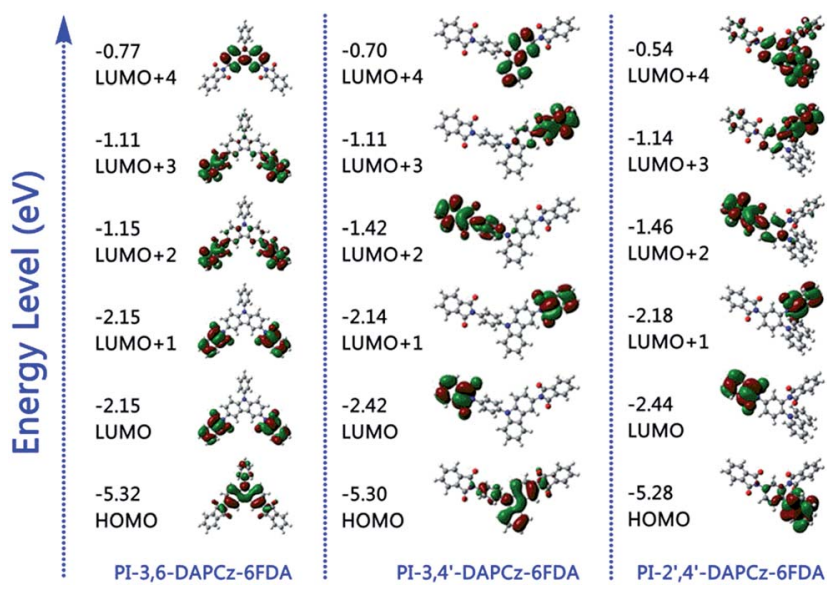

Fig. 8 Calculated molecular orbitals and corresponding energy levels of the basic units of synthesized isomeric Pls. overlapping zone of the two orbitals. Excitation of the donor leads to a decrease in ionization potential and hence promotes intramolecular CT at the excited state. The excited electrons at the LUMO+4 are energetic and prone to relax to the degenerate $\mathrm{LUMO}+2$ and LUMO+3, and finally to the degenerate LUMO+1 and LUMO, forming the CT complex (CTC) through internal conversion. Once after the formation of the CTC under external electric field, the holes resulting from the excitation of electrons at HOMO orbital can delocalize to the DAPCz moieties and generate an open channel, through which the charge carriers can migrate. As a result, the current instantly leap to the conductive state, switching the device to the ON state. The conductive CT states will be beneficial to the electrical conducting of both positive and negative direction voltages. Therefore, the device can't be switched off when a reverse bias scan is applying.

Mulliken charge population analysis provides theoretical proofs for the above CT process. Table 2 displays the Mulliken charges of the three isomeric polyimides in ground state and excited state. The $N$-phenylcarbazole moiety acts as the electron donor and the two phthalimides act as the acceptor, respectively. For ease of discussion, the acceptor was divided into two parts, acceptor 1 and acceptor 2 . As can be seen, the total charge on the donor part of PI-3,6-DAPCz-6FDA clearly becomes more positive (i.e., from 0.7051 to 0.8189 ) after excitation. And the negative charges on acceptor 1 and acceptor 2 are examined to become more negative and increase from $-0.3526,-0.3526$ to $-0.4095,-0.4095$, respectively. The charge variations observed on the donor and acceptor provides direct evidence for the proposed CT process.

However, distinct memory behaviors of the isomeric polyimides were observed after being transited to the ON state, i.e., SRAM with a retention time of $1 \mathrm{~min}$ for PI-3,6-DAPCz-6FDA, SRAM with a retention time of $5 \mathrm{~min}$ for PI-3,4'-DAPCz-6FDA and WORM for PI-2', $4^{\prime}$-DAPCz-6FDA. We figured that the spatial configuration might play a crucial role in the variation of the memory behaviors.

Fig. 9 shows the calculated favorable geometries and dihedral angles between aromatic components of the isomeric PIs. As observed, isomerization has significantly altered the conjugated molecular backbone of the electroactive PIs. It is suggested that the dihedral angles between adjoining rings along the conjugated molecular backbone possess definitive influence on the fluidity of the electrons, ${ }^{26}$ which will then affect the charge transfer process considerably. It is clear that the PI-3,6-DAPCz-

Table 2 Mulliken charges of the donor and acceptors in model compounds of the three isomeric polyimides

Mulliken charge

Ground state

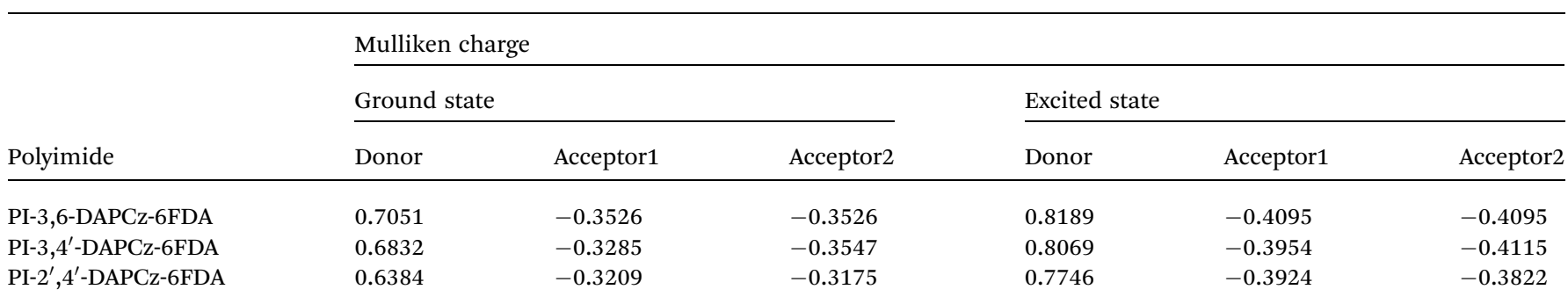




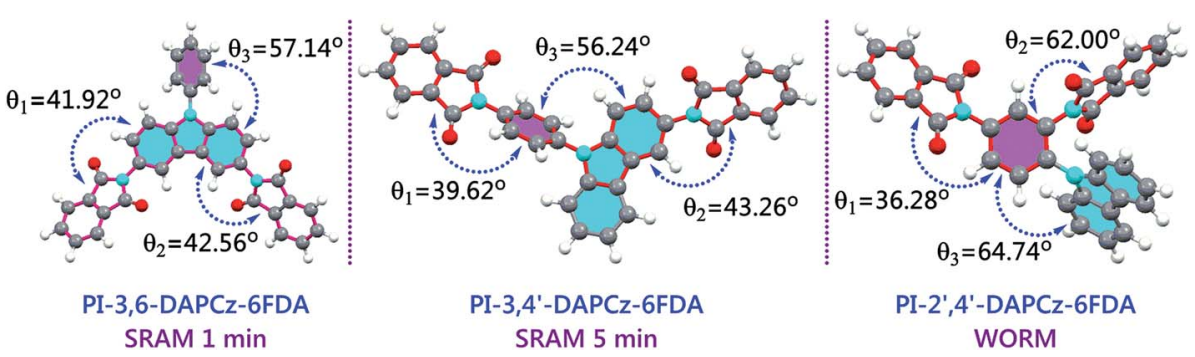

Fig. 9 Optimized geometries and dihedral angles between aromatic components of the isomeric polyimides. The chains in red represent for the backbone of the polyimides. The $\theta_{1}$ and $\theta_{2}$ are the dihedral angles between phthalimides and adjoining aromatic moieties. The $\theta_{3}$ is the dihedral angle between the phenyl ring and the carbazole moiety.

6FDA has a symmetrical structure with the carbazole unit entirely enclosed in the backbone. As illustrated in Fig. 8, the HOMO orbital locates mainly on the carbazole group and LUMO orbitals mainly locate on the phthalimide. Consequently, the main CT process will occur along the phthalimide group and carbazole group in the main chain. Thus, the CT process will not be affected significantly by the large dihedral angle between phenyl group and carbazole group $\left(\theta_{3}=57.14^{\circ}\right)$. And the relatively coplanar structure of the molecular backbone resulted from the relatively small dihedral angles of $\theta_{1}\left(42.56^{\circ}\right)$ and $\theta_{2}\left(41.92^{\circ}\right)$ will facilitate the charge CT and back CT process and therefore manifest a volatile SRAM type memory effect. In contrast, the PI3,4'-DAPCz-6FDA shows a much more twisted backbone structure. The phenyl group in DAPCz was fully enclosed, but the carbazole unit was only partially contained in the main chain. The CT and back CT process were considered to become much more difficult because of the large dihedral angle between the phenyl and carbazole group $\left(\theta_{3}=56.24^{\circ}\right)$, leading to a SRAM memory behavior with an extended retention time of $5 \mathrm{~min}$.

For the PI-2', 4'-DAPCz-6FDA, it is shown that only the phenyl group in DAPCz was enclosed in the backbone, and the carbazole unit was completely excluded and present as a pendant group. Obviously, the large dihedral angle between phenyl moiety and phthalimide group $\left(\theta_{2}=62.00^{\circ}\right)$ and the large dihedral angle between carbazole group and phenyl group $\left(\theta_{3}=\right.$ $64.74^{\circ}$ ) will produce considerably high energy barrier for the CT process to overcome. ${ }^{9,21}$ Hence, the PI-2', $4^{\prime}$-DAPCz-6FDA device exhibits the highest switching-on voltage $(3.0 \mathrm{~V}$ and $-3.0 \mathrm{~V})$ among the three isomeric PI devices. And, the back CT process and the disassociation of the formed CTC will be severely prohibited after power off, because of the large dihedral angle between the pendant electron donor and the backbone $\left(\theta_{3}=\right.$ $64.74^{\circ}$ ), and the huge steric hindrance between carbazole group and adjacent phthalimide. This fact will accordingly results in the irreversibility of the CTC and the non-volatile WORM feature of the PI-2' ${ }^{\prime} 4^{\prime}$-DAPCz-6FDA.

\section{Conclusion}

In summary, three isomeric PIs, PI-3,6-DAPCz-6FDA, PI-3,4'DAPCz-6FDA and PI-2' ${ }^{\prime}{ }^{\prime}$-DAPCz-6FDA have been successfully synthesized and demonstrated to show the volatile SRAM (retention time: $1 \mathrm{~min}$ ), SRAM (retention time: $5 \mathrm{~min}$ ), and non- volatile WORM behavior, respectively. Isomerization has significantly altered the conjugated molecular backbone of the electroactive PIs. The PI-3,6-DAPCz-6FDA possesses the best structural coplanarity, which greatly facilitates its CT and back CT process, leading to the volatile SRAM feature. The backbone structure of the PI-3,4'-Cz-6FDA becomes rather twisted, which consequently hinders the charge transition along the main chain, resulting in a SRAM effect with extended retention time. In contrast, the PI-2' $4^{\prime}$-DAPCz-6FDA exhibits vastly different backbone structure, where the primary electron-donating unit (carbazole units) are excluded from the conjugated main chain and present as a pendant. The considerably large dihedral angle between the pendant electron donor and the backbone hinders the CT process and prohibits the back CT process, accounting for its non-volatile WORM behavior. Besides, optical measurements indicate the formation of more stable CT complexes in the PI-2', $4^{\prime}$-DAPCz-6FDA than those in the PI-3,6-DAPCz-6FDA and PI-3,4'-Cz-6FDA, consistent with their non-volatile and volatile memory characteristics. The memory devices derived from these isomeric PIs all possess excellent long-term operation stability and ultrafast response time. The present research provides a novel strategy on designing the functional PIs for high-performance memory device application.

\section{Acknowledgements}

The authors sincerely appreciate the financial support from the National Basic Research Program of China (973 Program, 2014CB643604), National Natural Science Foundation of China (51673017, 21404005, 51273018), the Foundation Research Project of Jiangsu (Natural Science Foundation for Distinguished Young Scholars, BK20140006, Natural Science Foundation, BK20130256 and BK20150273), Changzhou Sci \& Tech Program (CZ20150001), as well as the support from CHEMCLOUDCOMPUTING@BUCT.

\section{References}

1 Q. D. Ling, D. J. Liaw, E. Y. H. Teo, C. Zhu, D. S. H. Chan, E. T. Kang and K. G. Neoh, Polymer, 2007, 48, 5182.

2 Q. D. Ling, D. J. Liaw, C. Zhu, D. S. H. Chan, E. T. Kang and K. G. Neoh, Prog. Polym. Sci., 2008, 33, 917. 
3 S. Song, B. Cho, T. W. Kim, Y. Ji, M. Jo, G. Wang, M. Choe, Y. H. Kahng, H. Hwang and T. Lee, Adv. Mater., 2010, 22, 5048.

4 T. Kurosawa, T. Higashihara and M. Ueda, Polym. Chem., 2013, 4, 16.

5 Q. D. Ling, F. C. Chang, Y. Song, C. X. Zhu, D. J. Liaw, D. S. H. Chan, E. T. Kang and K. G. Neoh, J. Am. Chem. Soc., 2006, 128, 8732.

6 S. G. Hahm, S. Choi, S. H. Hong, T. J. Lee, S. Park, D. M. Kim, W. S. Kwon, K. Kim, O. Kim and M. Ree, Adv. Funct. Mater., 2008, 18, 3276.

7 S. G. Hahm, S. Choi, S. H. Hong, T. J. Lee, S. Park, D. M. Kim, J. C. Kim, W. Kwon, K. Kim, M.-J. Kim, O. Kim and M. Ree, J. Mater. Chem., 2009, 19, 2207.

8 D. M. Kim, S. Park, T. J. Lee, S. G. Hahm, K. Kim, J. C. Kim, W. Kwon and M. Ree, Langmuir, 2009, 25, 11713.

9 T. Kuorosawa, C. C. Chueh, C. L. Liu, T. Higashihara, M. Ueda and W. C. Chen, Macromolecules, 2010, 43, 1236.

10 Q. Liu, K. Jiang, Y. Wen, J. Wang, J. Luo and Y. Song, Appl. Phys. Lett., 2010, 97, 253304.

11 Y. Li, R. Fang, S. Ding and Y. Shen, Macromol. Chem. Phys., 2011, 212, 2360.

12 Y. Q. Li, R. C. Fang, A. M. Zheng, Y. Y. Chu, X. Tao, H. H. Xu, S. J. Ding and Y. Z. Shen, J. Mater. Chem., 2011, 21, 15643.

13 C. J. Chen, H. J. Yen, W. C. Chen and G. S. Liou, J. Mater. Chem., 2012, 22, 14085.

14 Y. H. Chou, N. H. You, T. Kurosawa, W. Y. Lee, T. Higashihara, M. Ueda and W. C. Chen, Macromolecules, 2012, 45, 6946.
15 Y. Liu, Y. Zhang, Q. Lan, S. Liu, Z. Qin, L. Chen, C. Zhao, Z. Chi, J. Xu and J. Economy, Chem. Mater., 2012, 24, 1212.

16 T. Kurosawa, Y. C. Lai, A. D. Yu, H. C. Wu, T. Higashihara, M. Ueda and W. C. Chen, J. Polym. Sci., Part A: Polym. Chem., 2013, 51, 1348.

17 B. J. Ree, W. Kwon, K. Kim, Y. G. Ko, Y. Y. Kim, H. Lee and M. Ree, ACS Appl. Mater. Interfaces, 2014, 6, 21692.

18 L. Dong, H. S. Sun, J. T. Wang, W. Y. Lee and W. C. Chen, J. Polym. Sci., Part A: Polym. Chem., 2015, 53, 602.

19 C. L. Tsai, K. Sudhir K Reddy, C. Y. Yeh, C. L. Wang, C. Y. Lin, H. J. Yen, M. C. Tsai and G. S. Liou, RSC Adv., 2016, 6, 88531.

20 C. J. Chen, H. J. Yen, W. C. Chen and G. S. Liou, J. Polym. Sci., Part A: Polym. Chem., 2011, 49, 3709.

21 Y. L. Liu, K. L. Wang, G. S. Huang, C. X. Zhu, E. S. Tok, K. G. Neoh and E. T. Kang, Chem. Mater., 2009, 21, 3391.

22 Y. G. Ko, W. Kwon, H. J. Yen, C. W. Chang, D. M. Kim, K. Kim, S. G. Hahm, T. J. Lee, G. S. Liou and M. Ree, Macromolecules, 2012, 45, 3749.

23 G. Tian, S. Qi, F. Chen, L. Shi, W. Hu and D. Wu, Appl. Phys. Lett., 2011, 98.

24 B. Hu, F. Zhuge, X. Zhu, S. Peng, X. Chen, L. Pan, Q. Yan and R. W. Li, J. Mater. Chem., 2012, 22, 520.

25 M. C. Tsai, C. L. Wang, C. Y. Lin, C. L. Tsai, H. J. Yen, H. C. You and G. S. Liou, Polym. Chem., 2016, 7, 2780.

26 I. Cacelli, A. Feretti, M. Girlanda and M. Macucci, Chem. Phys., 2006, 320, 84. 